\title{
Ray equations of a weak shock in a hyperbolic system of conservation laws in multi-dimensions
}

\author{
PHOOLAN PRASAD \\ Department of Mathematics, Indian Institute of Science, Bangalore 560 012, India \\ E-mail: prasad@math.iisc.ernet.in
}

MS received 1 August 2014; revised 29 July 2015

\begin{abstract}
In this paper we give a complete proof of a theorem, which states that 'for a weak shock, the shock ray velocity is equal to the mean of the ray velocities of nonlinear wavefronts just ahead and just behind the shock, provided we take the wavefronts ahead and behind to be instantaneously coincident with the shock front. Similarly, the rate of turning of the shock front is also equal to the mean of the rates of turning of such wavefronts just ahead and just behind the shock'. A particular case of this theorem for shock propagation in gasdynamics has been used extensively in applications. Since it is useful also in other physical systems, we present here the theorem in its most general form.
\end{abstract}

Keywords. Ray theory; nonlinear waves; conservation laws; shock propagation and weak curved shock.

Mathematics Subject Classification. 35L02, 35L40, 35L60, 35L65, 35L67, 58J45, $74 \mathrm{~J} 40$.

\section{Introduction}

The simplest example of the result, which we take up to prove here, is seen in the case of the conservation law $u_{t}+\left(\frac{1}{2} u^{2}\right)_{x}=0$ from the Burgers' equation $u_{t}+u u_{x}=0$. The well known result for this equation says that the shock velocity $S$ is the mean of the characteristic velocities $u_{1}$ and $u_{\mathrm{r}}$ respectively on the left and on the right of the shock, i.e., $S=\frac{1}{2}\left(u_{1}+u_{\mathrm{r}}\right)$. As in this case, the subscripts 'l' and 'r' on a quantity will denote the values of the quantity just on the left and just on the right of a shock under consideration, through out this paper.

The above result is true approximately for a weak shock in the solution of a hyperbolic system of $n$ conservation laws in one space dimension ([2] and Theorem 17.16a of [9]). For such a system, the eigenvalues can be arranged in the form $c_{1} \leq c_{2} \leq \cdots \leq c_{n}$. A wave moving with a velocity $c_{k}$ is referred to a wave in $k$-th characteristic field, which may either be linearly degenerate or genuinely nonlinear [9]. Let $S$ be the shock velocity of a shock in the $k$-th genuinely nonlinear characteristic field with characteristic velocity $c$ (after dropping the subscript $k$ from $c$ ) and let the strength of the shock be denoted by $\varepsilon>0$, then

$$
S=\frac{1}{2}\left(c_{1}+c_{\mathrm{r}}\right)+O\left(\varepsilon^{2}\right), \text { for } \varepsilon \ll 1 .
$$


We shall extend the result (1.1) to a hyperbolic system of $n$ conservation laws in multidimensions $(\mathbf{x}, t)$, where $\mathbf{x}=\left(x_{1}, x_{2}, \ldots, x_{d}\right) \in \mathbb{R}^{d}$ :

$$
\mathbf{H}_{\mathbf{t}}(\mathbf{u})+\mathbf{F}_{x_{1}}^{(1)}(\mathbf{u})+\mathbf{F}_{x_{2}}^{(2)}(\mathbf{u})+\cdots+\mathbf{F}_{x_{d}}^{(d)}(\mathbf{u})=0,
$$

where $\mathbf{u} \in \mathbb{R}^{n}, \mathbf{H} \in \mathbb{R}^{n}$ and $\mathbf{F}^{(\alpha)} \in \mathbb{R}^{n}$. We assume that $\mathbf{H}$ and $\mathbf{F}^{(\alpha)}$ are smooth. The differential form of this system of conservation laws is a hyperbolic system of partial differential equations

$$
A(\mathbf{u}) \mathbf{u}_{t}+B^{(\alpha)}(\mathbf{u}) \mathbf{u}_{x_{\alpha}}=0,
$$

where

$$
A(\mathbf{u})=\left\langle\nabla_{\mathbf{u}}, \mathbf{H}\right\rangle \text { and } B^{(\alpha)}(\mathbf{u})=\left\langle\nabla_{\mathbf{u}}, \mathbf{F}^{(\alpha)}\right\rangle,
$$

and we use the summation convention that a repeated symbol in subscripts and superscripts in a term will mean summation over the range of the symbol. The range of the symbols $\alpha, \beta$, and $\gamma$ will be $1,2, \ldots, d$ and those of $i, j$, and $k$ will be $1,2, \ldots, n$.

In one dimension (one space dimension), a nonlinear wavefront and a shock front are points moving with time and these points are projections of sections by $t=$ constant respectively of a characteristic curve $\varphi(x, t)=0$ and a shock curve $\mathcal{S}(x, t)=0$ in the $(x, t)$-plane. In $d$-space-dimensions a nonlinear wavefront is the projection on the $\mathbf{x}$-space of a section by $t=$ constant of a characteristic surface $\varphi(\mathbf{x}, t)=0$ in space-time and similarly a shock front (or simply a shock) is the projection of a section by $t=$ constant of shock manifold $\mathcal{S}(\mathbf{x}, t)=0$. In $\S 2$, we shall write the ray equations of a nonlinear wavefront. In that section, we shall also introduce the shock manifold equation (SME), which is a partial differential equation satisfied by $\mathcal{S}(\mathbf{x}, t)$. In $\S 3$, we shall deduce the relation between the ray equations of the nonlinear wavefront and the shock ray equations derived from the SME.

We take a known solution $\mathbf{u}(\mathbf{x}, t)$ (smooth except for a jump discontinuity on a shock manifold $\mathcal{S}(\mathbf{x}, t)=0$ ) for which $A$ and $B^{(\alpha)}$ become known functions of $\mathbf{x}$ and $t$.

\section{Ray equations of a nonlinear wavefront and a shock front}

The unit normal $\mathbf{n}$ of a nonlinear wavefront $\Omega_{t}: \varphi(\mathbf{x}, t)=0, t=$ constant, and its (normal) velocity $c$ are given by

$$
\mathbf{n}=\frac{\nabla \varphi}{|\nabla \varphi|} \quad \text { and } \quad c=-\frac{\varphi_{t}}{|\nabla \varphi|} .
$$

The velocity $c$ of the wavefront is an eigenvalue of the system (1.3) and satisfies the characteristic equation

$$
\operatorname{det}\left[n_{\alpha} B^{(\alpha)}-c A\right]=0 .
$$

We assume that $c$ is a simple root of (2.2). Let the left and right eigenvectors of the characteristic matrix in (2.2) be denoted respectively by $\boldsymbol{l}$ and $\mathbf{r}$. They satisfy

$$
\mathbf{l} n_{\alpha} B^{(\alpha)}=c \mathbf{l} A, \quad n_{\alpha} B^{(\alpha)} \mathbf{r}=c A \mathbf{r} .
$$

In space-time, $\varphi(\mathbf{x}, t)=0$ represents a $d$-dimensional characteristic surface $\Omega$, which is generated by a $d-1$ parameter family of bicharacteristic curves on it (page 69 of [6]). 
Projection of the tangent direction of a bicharacteristic on $\mathbf{x}$-space gives the ray velocity direction associated with the wavefront $\Omega_{t}$. We denote the ray velocity by $\chi$. We state here the lemma on bicharacteristics directions, which was first proved using geometric concepts in [1]. A complete non-geometric proof of this lemma is also possible.

Lemma 2.1. The components $\chi_{\alpha}$ of the ray velocity $\chi$ corresponding to the eigenvalue $c$ are $\chi_{\alpha}=\frac{1 B^{(\alpha)} \mathbf{r}}{1 A \mathbf{r}}$, and hence along a nonlinear ray

$$
\frac{\mathrm{d} x_{\alpha}}{\mathrm{d} t}=\frac{\mathbf{l} B^{(\alpha)} \mathbf{r}}{\mathbf{l} A \mathbf{r}}=: \chi_{\alpha}, \quad \alpha=1,2, \ldots, d
$$

We take the first relation in (2.3) and post-multiply by $\mathbf{r}$. This gives wavefront velocity $c=\langle\mathbf{n}, \chi\rangle$. We also use $n_{\alpha}=\varphi_{x_{\alpha}} /|\nabla \varphi|$ and $c=-\varphi_{t} /|\nabla \varphi|$ and get an eikonal equation for the nonlinear wavefront

$$
Q\left(\varphi_{t}, \nabla \varphi\right):=(\mathbf{l} A \mathbf{r}) \varphi_{t}+\left(\mathbf{l} B^{(\alpha)} \mathbf{r}\right) \varphi_{x_{\alpha}}=0 .
$$

Since $\mathbf{l}$ and $\mathbf{r}$ depend on $\mathbf{n}$, this equation is a first order nonlinear PDE in $\varphi$.

Remark 2.2. Take a general $\chi$, not necessarily given by (2.4) and consider the $\operatorname{PDE} \varphi_{t}+$ $\chi_{\alpha} \varphi_{x_{\alpha}}=0$. We shall be tempted to conclude that $\chi$ is a ray velocity according to which the surface $\varphi(\mathbf{x}, t)=0, t=$ constant, evolves. However, in order that $\chi$ qualifies to be a ray velocity, is must satisfy a consistency condition

$$
n_{\beta} n_{\gamma}\left(n_{\beta} \frac{\partial}{\partial n_{\alpha}}-n_{\alpha} \frac{\partial}{\partial n_{\beta}}\right) \chi_{\gamma}=0, \quad \text { for each } \alpha=1,2, \ldots, d .
$$

A proof is available in [7]. It has also been proved there that the ray velocity with components $\chi_{\alpha}=\frac{1 B^{(\alpha)} \mathbf{r}}{1 A \mathbf{r}}$ of a hyperbolic system does satisfy (2.6).

The ray equation (2.4) is not complete, since we need an equation for another unknown $\mathbf{n}$ appearing in $\mathbf{I}$ and $\mathbf{r}$. The equation for $\mathbf{n}$ was first derived in [5] (see also [6] and [7]) in the form

$$
\frac{\mathrm{d} n_{\alpha}}{\mathrm{d} t}=-\frac{1}{\mathbf{I} A \mathbf{r}} \mathbf{l}\left\{n_{\beta}\left(n_{\gamma} \frac{\partial B^{(\gamma)}}{\partial \eta_{\beta}^{\alpha}}-c \frac{\partial A}{\partial \eta_{\beta}^{\alpha}}\right)\right\} \mathbf{r}=\psi_{\alpha}, \text { say, }
$$

where we note that

$$
\frac{\mathrm{d}}{\mathrm{d} t}=\frac{\partial}{\partial t}+\chi_{\alpha} \frac{\partial}{\partial x_{\alpha}}, \quad \frac{\partial}{\partial \eta_{\beta}^{\alpha}}=n_{\beta} \frac{\partial}{\partial x_{\alpha}}-n_{\alpha} \frac{\partial}{\partial x_{\beta}} .
$$

The system of equations (2.4) and (2.7) are bicharacteristic or ray equations of a nonlinear wavefront in the chosen $k$-th characteristic field.

Now, we proceed to derive the shock ray equations but we need the eikonal equation of a shock, i.e., the shock manifold partial differential equation (SME) $[4,6]$ in the $(\mathbf{x}, t)$ space. The jump relation or RH condition across a shock $\mathcal{S}(\mathbf{x}, t)=0$ for the system of conservation laws, with the well known notation $[g]$ for the jump $g\left(\mathbf{u}_{1}\right)-g\left(\mathbf{u}_{\mathrm{r}}\right)$, is

$$
\mathcal{S}_{t}[\mathbf{H}]+\mathcal{S}_{x_{\alpha}}\left[\mathbf{F}^{\alpha}\right]=\mathbf{0}
$$


or, in terms of the velocity $S$ and unit normal $\mathbf{N}$ of the shock, given by

$$
S=-\mathcal{S}_{t} /\left|\nabla_{\mathbf{x}} \mathcal{S}\right| \quad \text { and } \quad \mathbf{N}=\nabla_{\mathbf{x}} \mathcal{S} /\left|\nabla_{\mathbf{x}} \mathcal{S}\right|
$$

the jump relation becomes

$$
-S[\mathbf{H}]+N_{\alpha}\left[\mathbf{F}^{\alpha}\right]=\mathbf{0} .
$$

The jump relation (2.9) is a system of $n$ relations and we can derive (as shown in [4]) a large number of scalar relations (containing components of $\mathbf{u}_{1}$ and $\mathbf{u}_{\mathrm{r}}$ ) which would qualify for being a SME . Let us discuss here taking just one such relation. As the functions $\mathbf{u}_{1}$ and $\mathbf{u}_{\mathrm{r}}$ are defined only in the domains to the left and to the right of the shock, this relation would be valid only on the shock manifold $\mathcal{S}(\mathbf{x}, t)=0$ and not in neighourhood. Hence it cannot be treated as a PDE in $(\mathbf{x}, t)$-space. Assuming that $\mathbf{u}_{1}$ and $\mathbf{u}_{\mathrm{r}}$ are smooth in their respective domains, we extend them on the other side of the shock as smooth functions. Now the relation would become a PDE [4]. Shock rays first appeared in the work of Maslov [3] and the fact that non-unique extension of functions as smooth functions on the other side of the shock does not effect the uniqueness of shock rays has been argued in [4].

\section{Proof of the theorem on weak shock}

Existence of more than one shock manifold equations (SMEs) and the question of uniqueness of shock rays for Euler equations have been discussed in [4]. Results for a weak shock have also been obtained there. We state a theorem in the most general form for the system of conservation laws (1.2).

Theorem 3.1. For a weak shock, the shock ray velocity is equal to the mean of the ray velocities of the nonlinear wavefronts just ahead and just behind the shock, provided we take the wavefronts ahead and behind to be instantaneously coincident with the shock front. Similarly, the rate of turning of the shock front, i.e., $\mathrm{d} \mathbf{N} / \mathrm{d} t$, is also equal to the mean of the rates of turning of such wavefronts just ahead and just behind the shock.

These are approximate results correct up to first order in the shock strength.

Remark 3.2. We note that the nonlinear waves of the $k$-th family, on the two sides of the shock, need not be of small amplitude.

Proof. We shall find an approximate form of the jump relations (2.9) of the system of conservation laws (1.2) assuming the shock strength $:=\left|\mathbf{u}_{1}-\mathbf{u}_{\mathrm{r}}\right|=\varepsilon$ to be small. We first note that

$$
\mathbf{H}\left(\frac{1}{2}\left(\mathbf{u}_{1}+\mathbf{u}_{\mathrm{r}}\right)\right)=\mathbf{H}\left(\mathbf{u}_{1}\right)-\frac{1}{2}\left\langle\left\langle\nabla_{\mathbf{u}}, \mathbf{H}\right\rangle\left(\mathbf{u}_{1}\right),\left(\mathbf{u}_{1}-\mathbf{u}_{\mathrm{r}}\right)\right\rangle+O\left(\varepsilon^{2}\right)
$$

and

$$
\mathbf{H}\left(\frac{1}{2}\left(\mathbf{u}_{1}+\mathbf{u}_{\mathrm{r}}\right)\right)=\mathbf{H}\left(\mathbf{u}_{\mathrm{r}}\right)+\frac{1}{2}\left\langle\left\langle\nabla_{\mathbf{u}}, \mathbf{H}\right\rangle\left(\mathbf{u}_{\mathrm{r}}\right),\left(\mathbf{u}_{1}-\mathbf{u}_{\mathrm{r}}\right)\right\rangle+O\left(\varepsilon^{2}\right) .
$$


Subtracting and noting $A(\mathbf{u})=\left\langle\nabla_{\mathbf{u}}, \mathbf{H}\right\rangle$ and $B^{(\alpha)}(\mathbf{u})=\left\langle\nabla_{\mathbf{u}}, \mathbf{F}^{(\alpha)}\right\rangle$, we get

$$
[\mathbf{H}]=\left(\frac{1}{2}\left(A\left(\mathbf{u}_{1}\right)+A\left(\mathbf{u}_{\mathrm{r}}\right)\right)\right)\left(\mathbf{u}_{1}-\mathbf{u}_{\mathrm{r}}\right)+O\left(\varepsilon^{2}\right) .
$$

Similarly, we write approximations for $\mathbf{F}^{(\alpha)}\left(\frac{1}{2}\left(\mathbf{u}_{1}+\mathbf{u}_{\mathrm{r}}\right)\right), \alpha=1, \ldots, d$, and deduce expressions for $\left[\mathbf{F}^{(\alpha)}\right]$.

Substituting the above approximate relations in (2.9), and retaining only the leading order terms in $\left|\mathbf{u}_{1}-\mathbf{u}_{\mathrm{r}}\right|=\varepsilon$, we get

$$
\left\{\mathcal{S}_{t}\left(\frac{1}{2}\left(A\left(\mathbf{u}_{1}\right)+A\left(\mathbf{u}_{\mathrm{r}}\right)\right)\right)+\mathcal{S}_{x_{\alpha}}\left(\frac{1}{2}\left(B^{(\alpha)}\left(\mathbf{u}_{1}\right)+B^{(\alpha)}\left(\mathbf{u}_{\mathrm{r}}\right)\right)\right\}\left(\mathbf{u}_{1}-\mathbf{u}_{\mathrm{r}}\right)=\mathbf{0} .\right.
$$

For a shock $\mathbf{u}_{1}-\mathbf{u}_{\mathrm{r}} \neq \mathbf{0}$. Let the approximate shock velocity corresponding to the approximate values of $[\mathbf{H}]$ and $\left[\mathbf{F}^{(\alpha)}\right]$ as given in (3.3) etc. be $S_{1 \mathrm{r}}$. Then the matrix

$$
M_{\mathrm{lr}}:=-\left(\frac{1}{2}\left(A\left(\mathbf{u}_{1}\right)+A\left(\mathbf{u}_{\mathrm{r}}\right)\right)\right) S_{\mathrm{lr}}+\left(\frac{1}{2} B^{(\alpha)}\left(\mathbf{u}_{1}\right)+\frac{1}{2} B^{(\alpha)}\left(\mathbf{u}_{\mathrm{r}}\right)\right) N_{\alpha}
$$

is singular. Let $\mathbf{L}_{\mathrm{lr}}$ and $\mathbf{R}_{\mathrm{lr}}$ be the left and right eigenvectors of the matrix $M_{\mathrm{lr}}$.

From (3.4) we get an approximate value of the jump in $\mathbf{u}$,

$$
\mathbf{u}_{1}-\mathbf{u}_{\mathrm{r}}=\mathbf{R}_{\mathrm{lr}} W, \quad W \neq 0,
$$

where $W=O(\varepsilon)$ is a measure of the jump in $\mathbf{u}$ and hence a measure of the strength of the shock. Substituting (3.6) in (3.4), premultiplying by $\mathbf{L}_{\mathrm{lr}}$ and dividing by $\frac{1}{2} \mathbf{L}_{\mathrm{lr}}\left(\left(A\left(\mathbf{u}_{l}\right)+\right.\right.$ $\left.A\left(\mathbf{u}_{\mathrm{r}}\right)\right) \mathbf{R}_{\mathrm{lr}} W$, we get a scalar result

$$
\mathcal{S}_{t}+\left\{\frac{1}{2}\left(\frac{\mathbf{L}_{\mathrm{lr}} B^{(\alpha)}\left(\mathbf{u}_{1}\right) \mathbf{R}_{\mathrm{lr}}}{\frac{1}{2} \mathbf{L}_{\mathrm{lr}}\left(\left(A\left(\mathbf{u}_{1}\right)+A\left(\mathbf{u}_{\mathrm{r}}\right)\right) \mathbf{R}_{\mathrm{lr}}\right.}\right)+\frac{1}{2}\left(\frac{\mathbf{L}_{\mathrm{lr}} B^{(\alpha)}\left(\mathbf{u}_{\mathrm{r}}\right) \mathbf{R}_{\mathrm{lr}}}{\frac{1}{2} \mathbf{L}_{\mathrm{lr}}\left(\left(A\left(\mathbf{u}_{1}\right)+A\left(\mathbf{u}_{\mathrm{r}}\right)\right) \mathbf{R}_{\mathrm{lr}}\right.}\right)\right\} \mathcal{S}_{x_{\alpha}}=0,
$$

which is an approximate form of (2.9) correct up to first power of $\varepsilon$.

Let $c_{l}$ and $c_{r}$ be the velocities of the nonlinear wavefronts just behind and just ahead of the shock front $S_{t}: \mathcal{S}(\mathbf{x}, t)=0$ respectively and instantaneously coincident with it at time $t$. Let $\mathbf{l}_{1}$ and $\mathbf{r}_{1}$ be the left and right eigenvectors of the matrix $M_{1}:=-A\left(\mathbf{u}_{1}\right) c\left(\mathbf{u}_{1}\right)+$ $B^{(\alpha)}\left(\mathbf{u}_{1}\right) N_{\alpha}$. Similarly we define $\mathbf{l}_{\mathrm{r}}$ and $\mathbf{r}_{\mathrm{r}}$ as the left and right eigenvectors of the matrix $M_{\mathrm{r}}:=-A\left(\mathbf{u}_{\mathrm{r}}\right) c\left(\mathbf{u}_{\mathrm{r}}\right)+B^{(\alpha)}\left(\mathbf{u}_{\mathrm{r}}\right) N_{\alpha}$. Then it is easy to show that $\left|c_{1}-S\right|=O(\varepsilon)=\mid c_{r}$ $S \mid$. We also note

$$
\mathbf{L}_{1 \mathrm{r}}=\mathbf{l}_{1}+O(\varepsilon), \mathbf{L}_{\mathrm{lr}}=\mathbf{r}_{1}+O(\varepsilon), \mathbf{L}_{\mathrm{lr}}=\mathbf{l}_{\mathrm{r}}+O(\varepsilon), \mathbf{L}_{\mathrm{lr}}=\mathbf{r}_{\mathrm{r}}+O(\varepsilon) .
$$

Retaining only the most dominant terms, we can derive the following approximate results

$$
\frac{\mathbf{L}_{1 \mathrm{r}} B^{(\alpha)}\left(\mathbf{u}_{1}\right) \mathbf{R}_{\mathrm{lr}}}{\frac{1}{2} \mathbf{L}_{\mathrm{lr}}\left(\left(A\left(\mathbf{u}_{1}\right)+A\left(\mathbf{u}_{\mathrm{r}}\right)\right) \mathbf{R}_{\mathrm{lr}}\right.}=\frac{\mathbf{l}_{1} B^{(\alpha)}\left(\mathbf{u}_{1}\right) \mathbf{r}_{1}}{\mathbf{l}_{1} A\left(\mathbf{u}_{1}\right) \mathbf{r}_{1}}
$$


and

$$
\frac{\mathbf{L}_{\mathrm{lr}} B^{(\alpha)}\left(\mathbf{u}_{\mathrm{r}}\right) \mathbf{R}_{\mathrm{lr}}}{\frac{1}{2} \mathbf{L}_{\mathrm{lr}}\left(\left(A\left(\mathbf{u}_{1}\right)+A\left(\mathbf{u}_{\mathrm{r}}\right)\right) \mathbf{R}_{\mathrm{lr}}\right.}=\frac{\mathbf{l}_{\mathrm{r}} B^{(\alpha)}\left(\mathbf{u}_{\mathrm{r}}\right) \mathbf{r}_{\mathrm{r}}}{\mathbf{l}_{\mathrm{r}} A\left(\mathbf{u}_{\mathrm{r}}\right) \mathbf{r}_{\mathrm{r}}} .
$$

Finally, the approximate relation (3.7) is replaced by another approximate relation of (2.9) correct up to $O(\varepsilon)$ for a weak shock

$$
Q_{\mathrm{lr}}\left(t, \mathbf{x}, \mathcal{S}_{t}, \mathcal{S}_{x_{\alpha}}\right):=\mathcal{S}_{t}+\frac{1}{2}\left\{\frac{\mathbf{l}_{1} B^{(\alpha)}\left(\mathbf{u}_{1}\right) \mathbf{r}_{1}}{\mathbf{l}_{1} A\left(\mathbf{u}_{1}\right) \mathbf{r}_{1}}+\frac{\mathbf{l}_{\mathrm{r}} B^{(\alpha)}\left(\mathbf{u}_{\mathrm{r}}\right) \mathbf{r}_{\mathrm{r}}}{\mathbf{l}_{\mathrm{r}} A\left(\mathbf{u}_{\mathrm{r}}\right) \mathbf{r}_{\mathrm{r}}}\right\} \mathcal{S}_{x_{\alpha}}=0
$$

which we shall like to treat as a SME.

We emphasize a point already mentioned at the end of the last section. Since the functions $\mathbf{u}_{1}$ and $\mathbf{u}_{\mathrm{r}}$ are defined only in the domains to the left and to the right of the shock, the relation (3.10) is valid only on the shock manifold $\mathcal{S}(\mathbf{x}, t)=0$ and not in a neighbourhood of it. Hence it cannot be treated as a PDE. Assuming that they are smooth in their respective domains, we extend them on the other side of the shock as smooth functions. The relation (3.10) now becomes a PDE.

Note that $Q_{1}:=\mathcal{S}_{t}+\frac{\mathbf{l}_{1} B^{(\alpha)}\left(\mathbf{u}_{1}\right) \mathbf{r}_{1}}{\mathbf{l}_{1} A\left(\mathbf{u}_{1}\right) \mathbf{r}_{1}} \mathcal{S}_{x_{\alpha}}=0$ or $\mathcal{S}_{t}+\left(\chi_{\alpha}\right)_{1} \mathcal{S}_{x_{\alpha}}=0$ is the characteristic PDE (2.5) of the system (1.3) with $\mathbf{u}$ replaced by $\mathbf{u}_{1}$ and the normal $\mathbf{n}$ of the characteristic surface replaced by the normal $\mathbf{N}$ of the shock front. We can give a similar interpretation for the equation $Q_{\mathrm{r}}:=\mathcal{S}_{t}+\frac{\mathbf{l}_{\mathrm{r}} B^{(\alpha)}\left(\mathbf{u}_{\mathrm{r}}\right)_{1}}{\mathbf{l}_{\mathrm{r}} A\left(\mathbf{u}_{\mathrm{r}}\right) \mathbf{r}_{\mathrm{r}}} \mathcal{S}_{x_{\alpha}}=0$ or $\mathcal{S}_{t}+\left(\chi_{\alpha}\right)_{\mathrm{r}} \mathcal{S}_{x_{\alpha}}=0$.

Since the velocity with components $\frac{1 B^{(\alpha)} \mathbf{r}}{1 A \mathbf{r}}$ of a nonlinear wavefront does satisfy the consistency condition (2.6), the sum $\frac{\mathbf{l}_{1} B^{(\alpha)}\left(\mathbf{u}_{1}\right) \mathbf{r}_{1}}{\mathbf{l}_{1} A\left(\mathbf{u}_{1}\right) \mathbf{r}_{1}}+\frac{\mathbf{l}_{\mathbf{r}} B^{(\alpha)}\left(\mathbf{u}_{\mathrm{r}}\right) \mathbf{r}_{\mathbf{r}}}{\mathbf{l}_{\mathrm{r}} A\left(\mathbf{u}_{\mathrm{r}}\right) \mathbf{r}_{\mathrm{r}}}$ gives components of a velocity which also satisfies it with $\mathbf{n}$ replaced by $\mathbf{N}$ and does qualify to be a ray velocity. Now we deduce from (3.10) that

$$
\frac{\mathrm{d} X_{\alpha}}{\mathrm{d} t}:=\left(\frac{\mathrm{d} x_{\alpha}}{\mathrm{d} t}\right)_{\text {shock }}=\frac{1}{2}\left\{\left(\chi_{\alpha}\right)_{1}+\left(\chi_{\alpha}\right)_{\mathrm{r}}\right\}
$$

where $\mathbf{X}(t)$ is the position of the shock at time $t$. Since $\mathbf{u}_{1}$ and $\mathbf{N}$ appear in $\left(\chi_{\alpha}\right)_{1}$ in place of $\mathbf{u}$ and $\mathbf{n},\left(\chi_{\alpha}\right)_{1}$ is the ray velocity of the the system (1.4) for a nonlinear wavefront just on the left of the shock, provided we take the wavefront to be instantaneously coincident with the shock front. A similar interpretation follows for $\left(\chi_{\alpha}\right)_{\mathrm{r}}$. This equation proves the first part of Theorem 3.1. The shock ray velocity $\frac{\mathrm{d} X}{\mathrm{~d} t}$ is the mean of the ray velocities of nonlinear wavefronts just ahead and just behind the shock and instantaneously coincident with it.

We now give a derivation the second part of Theorem 3.1, namely

$$
\frac{\mathrm{d} N_{\alpha}}{\mathrm{d} t}=\frac{1}{2}\left\{\left(\psi_{\alpha}\right)_{1}+\left(\psi_{\alpha}\right)_{\mathrm{r}}\right\}
$$

where $\left(\psi_{\alpha}\right)_{1}$ and $\left(\psi_{\alpha}\right)_{\mathrm{r}}$ can be defined as $\left(\chi_{\alpha}\right)_{1}$ and $\left(\chi_{\alpha}\right)_{\mathrm{r}}$ above. More explicitly $\left(\psi_{\alpha}\right)_{1}$ is the expression $\psi_{\alpha}$ in (2.7) with $\mathbf{u}$ and $\mathbf{n}$ replaced by $\mathbf{u}_{1}$ and $\mathbf{N}$ respectively and represents the rate of turning of a nonlinear wavefront just behind the shock and coincident with it. A similar interpretation follows for $\left(\psi_{\alpha}\right)_{\mathrm{r}}$. The proof is very simple and follows from the second part of the ray equations of SME (3.10) as (2.7) follows from (2.5). 
The expressions for $\left(\psi_{\alpha}\right)_{1}$ and $\left(\psi_{\alpha}\right)_{\mathrm{r}}$, contain operators $\left(\frac{\partial}{\partial \eta_{\beta}^{\alpha}}\right)_{\text {shock }}$. We note that the operators $\frac{\mathrm{d}}{\mathrm{d} t}$ in (3.11) and (3.12) and $\left(\frac{\partial}{\partial \eta_{\beta}^{\alpha}}\right)_{\text {shock }}$ appearing in $\left(\psi_{\alpha}\right)_{1}$ and $\left(\psi_{\alpha}\right)_{\mathrm{r}}$ have explicit expressions:

$$
\begin{aligned}
\left(\frac{\mathrm{d}}{\mathrm{d} t}\right)_{\text {shock }} & =\frac{\partial}{\partial t}+\left(\frac{1}{2}\left\{\left(\chi_{\alpha}\right)_{1}+\left(\chi_{\alpha}\right)_{\mathrm{r}}\right\}\right) \frac{\partial}{\partial x_{\alpha}}, \\
\left(\frac{\partial}{\partial \eta_{\beta}^{\alpha}}\right)_{\text {shock }} & =N_{\beta} \frac{\partial}{\partial x_{\alpha}}-N_{\alpha} \frac{\partial}{\partial x_{\beta}} .
\end{aligned}
$$

\section{Conclusion}

Theorem 3.1 is for a curved weak shock in a $k$-th characteristic field. The theorem does not assume that the nonlinear waves on the two sides of the shock are of small amplitude. We have presented a very clear and complete proof of the theorem.

The problem of computing successive positions of a curved shock is extremely difficult. A particular case of the theorem derived for Euler equations of a polytropic gas for small amplitude nonlinear waves has been extensively used by us in many papers. By the use of the theorem we avoided difficult calculations of the expressions for $\frac{\mathrm{d} X_{\alpha}}{\mathrm{d} t}$ shock and $\frac{\mathrm{d} N_{\alpha}}{\mathrm{d} t}$ as seen in [8], where we could work out only in two space dimensions.

As mentioned in the previous paragraph, only a particular case of the theorem has been used when the nonlinear waves on the two sides are of small amplitude. But weak shocks also appear in a solution which is not a small amplitude perturbation on a given solution. Therefore, it is worthwhile using the theorem for tracing a curved weak shock fitted in nonlinear waves, which are not of small amplitude. One suggestion is to develop a hybrid numerical scheme for a hyperbolic system of conservation laws in which the weak shock is automatically fitted in the numerical solution with the help of this theorem. Weak shocks are diffused and hence tracing them is difficult in a numerical computation. Use of the theorem in a hybrid numerical scheme will give a sharp position and clear geometry of a weak shock.

\section{Acknowledgements}

This work is supported by the National Academy of Sciences, India under NASI Senior Scientist Platinum Jubilee Fellowship programme. The Department of Mathematics of IISc is partially supported by the UGC as a Centre of Advance Study and by DST through FIST Programme. The author would like to thank the referee, who went through the paper very carefully and suggested many improvements.

\section{References}

[1] Courant R and Hilbert D, Methods of Mathematical Physics, vol 2: Partial Differential Equations (1962) (Interscience Publishers)

[2] Lax P D, Hyperbolic system of conservation laws II, Comm. Pure Appl. Math. 10 (1957) 537-66 
[3] Maslov V P, Propagation of shock waves in an isentropic non-viscous gas, J. Sov. Math. 13 (1980) 119-163 (Russian publication 1978)

[4] Prasad P, Kinematics of a multi-dimensional shock of arbitrary strength in an ideal gas, Acta Mechanica 45 (1982) 163-76

[5] Prasad P, On the lemma on bicharacteristics, Appendix in: A nonlinear ray theory, Berichte der Arbeitsgruppe Technomathematik, Universitaet Kaiserslautern, Nr. 101 (1993)

[6] Prasad P, Nonlinear hyperbolic waves in multi-dimensions, Monographs and Surveys in Pure and Applied Mathematics, Chapman and Hall/CRC, 121 (2001)

[7] Prasad P, Ray theories for hyperbolic waves, kinematical conservation laws and applications, Indian J. Pure and Appl. Math. 38 (2007) 467-490

[8] Ravindran R and Prasad P, On infinite system of compatibility conditions along a shock ray, Q. J. Appl. Math. and Mech. 46 (1993) 131-140

[9] Smoller J, Shock waves and reaction diffusion equation (1983) (Springer-Verlag)

Communicating Editor: Meheboob Alam 